\section{Do I have enough time? The impact of recruiting patients to a randomised controlled trial at recruiting centres}

\author{
P. E. Ellis, ${ }^{1}$ R. L. Bradley, ${ }_{1}$ J. R. Sandy, ${ }_{1}^{3}$ S. A. Deacon, ${ }_{1}^{4}$ H. S. Griffiths ${ }_{1}^{5}$ \\ N. E. Atack, ${ }^{6}$ M. B. Moore, ${ }^{7}$ K. A. House, ${ }^{8}$ N. A. Wenger, ${ }_{1}^{9}$ V. Worth ${ }^{10}$ \\ and A. J. Ireland ${ }^{11}$
}

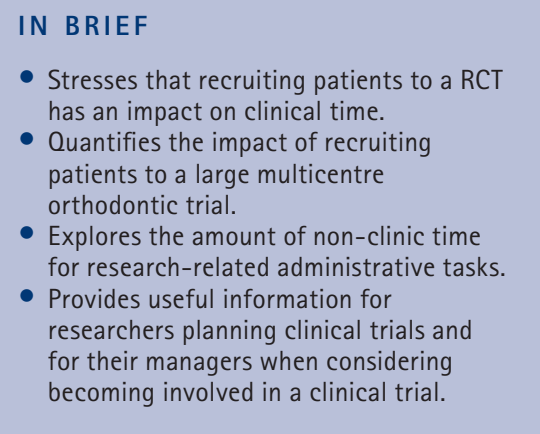

Introduction This paper explores the impact of recruiting patients to a randomised controlled trial (RCT) at recruiting centres. This large multicentre RCT examining the efficacy of chewing gum compared to ibuprofen in the relief of orthodontic pain was carried out across nine recruiting centres. Method The work diaries of clinicians and supporting staff at recruiting centres were analysed over a four-month period from September to December 2011. This quantified the amount of clinical and non-clinical time spent on research duties. Results Over this time period 98 patients were recruited across seven trial sites. On average, patient recruitment had a direct clinical impact of 19 minutes per patient recruited. The time commitment on trial administration outside the clinical sessions was much higher, averaging at 110 minutes per patient recruited, giving the overall time spent on the trial 129 minutes per patient. Conclusions This information will be valuable to lead researchers when calculating the full economic cost of a proposed clinical trial and therefore when applying for grant funding. It may also be valuable to clinicians and their managers when considering becoming a principle investigator (PI) in a RCT. Although the impact on clinical time was 19 minutes per patient recruited, there is a considerably higher (almost six times greater) time commitment in administration around the recruitment of patients.

\section{INTRODUCTION}

Multicentre RCTs are the gold standard for clinical research and the number of orthodontic multicentre RCTs has increased in the UK over the last ten years. Such trials have included diverse topics such as the correction of Class II malocclusions, ${ }^{1}$ orthodontic pain, ${ }^{2}$ the early correction of Class III malocclusions ${ }^{3}$ and self-ligating bracket systems. ${ }^{4}$

Recruiting sufficient patients into a study with adequate power to refute a defined null hypothesis is difficult. ${ }^{5}$ In order to increase recruitment to trials some orthodontic research has had to be extended beyond academic institutions into both primary and alternative secondary care settings. Previously, Hichens et al. ${ }^{6}$

1*-2Dorset County Hospital; ${ }^{3-4}$ Oral and Dental Science, Bristol; ${ }^{5}$ Yeovil District Hospital; ${ }^{6}$ Musgrove Park

Hospital, Taunton; ${ }^{7}$ Royal Devon and Exeter Hospital;

${ }^{8}$ Gloucestershire Hospitals NHS Foundation Trust;

${ }^{9}$ Royal Cornwall Hospital, Truro; ${ }^{10} \mathrm{Oral}$ and Dental Sci-

ence, Bristol; ${ }^{11}$ Royal United Hospitals, Bath

${ }^{*}$ Correspondence to: Mrs Pamela Ellis

Email: Pamela.Ellis@dchft.nhs.uk; Tel: 01305255768

\section{Refereed Paper}

Accepted 5 September 2012

DOI: 10.1038/sj.bdj.2012.986

${ }^{\circledR}$ British Dental Journal 2012; 2013: 467-470 examined the practical aspects of undertaking research in primary care and found that not only was it possible to recruit a large volume of patients in a relatively short time frame, but also that the results were more relevant since the data was collected in a setting which provides care for the majority of orthodontic patients. An alternative method of ensuring sufficient patient recruitment within a reasonable time frame is to recruit in a secondary care setting utilising a number of centres. Many district general hospitals have a large throughput of orthodontic patients, which makes them a good clinical setting for recruitment. In addition, many consultant orthodontists are keen to be involved in clinical research and indeed participation in research is an expectation of NHS trusts.

The aim of this paper is to examine the impact on workload at recruiting centres during the recruitment phase of a multicentre RCT.

\section{CLINICAL TRIAL BACKGROUND INFORMATION}

An RCT examining chewing gum and orthodontic pain commenced in November
2009. The primary aim of this study was to examine the efficacy of sugar-free chewing gum in the relief of orthodontic pain compared to ibuprofen. The secondary outcome measure was to determine whether sugar-free chewing gum increased orthodontic bracket or wire failure. In addition, patient anxiety was measured, as anxiety may affect the perception of pain.

One thousand patients aged 12-16 years were randomly allocated to the control group ( $2 \times 400 \mathrm{mg}$ ibuprofen as required) or the sugar-free chewing gum group (sugar-free chewing gum as required and ibuprofen $2 \times 400 \mathrm{mg}$ if the chewing gum was ineffective). Patients completed pain score charts in the three days following the fitting and then subsequent adjustment of orthodontic fixed appliances. At the fitting of the appliances clinicians gained consent for participation in the trial from patients' parents; confirmed allocation to the trial arm with the randomisation centre by telephone; recorded information about the appliances fitted; gave instructions to the patient on how to complete the pain score chart; recorded the patient's anxiety level; dispensed the 
ibuprofen \pm chewing gum; and completed the dispensing log. At the subsequent adjustment appointment clinicians recorded information on appliance breakages; recorded the patient's anxiety level; reinstructed patients on how to complete the second pain score chart; and dispensed further ibuprofen \pm chewing gum. In addition, clinicians recorded information about appliance breakages on the second adjustment appointment.

This was a non-commercial trial supported by funding from the British Orthodontic Society. The trial was included within the UK Clinical Research Network (UKCRN) portfolio database making it eligible for NHS service support costs. Ethics approval was gained from the North Somerset and South Bristol Research Ethics Committee. The trial was registered with the Medicines and Healthcare Products Regulatory Agency (MHRA).

The trial was coordinated by the chief investigator (CI) who had overall responsibility for the trial. The CI's duties included writing the protocol; gaining all of the necessary approvals including ethics, research and development (R\&D) and MHRA; securing funding; and recruiting trial sites and their principal investigators (PIs). The CI was also the PI for his trial site. There were nine trial sites in the South West of England including eight district general hospitals and one teaching hospital (Fig. 1). At each trial site the PI (a consultant orthodontist) was responsible for local organisation and day-to-day running of the trial. This included gaining local RED approval; compiling and updating site files; organising storage of drugs and dispensing logs with pharmacy; training other clinicians in the department in the recruitment of patients; recruiting patients; and ensuring the completion and submission of all paperwork.

The PIs at three of the sites received help in the recruitment of patients by their orthodontic colleagues. In addition, at three trial sites additional staff were available to help with recruitment. These were a research hygienist, an orthodontic nurse and an administrative assistant respectively.

At one trial site the PI took responsibility for administration and for the coordination of data collection and data entry. She was supported in this with administrative help funded by the trust's RetD department.

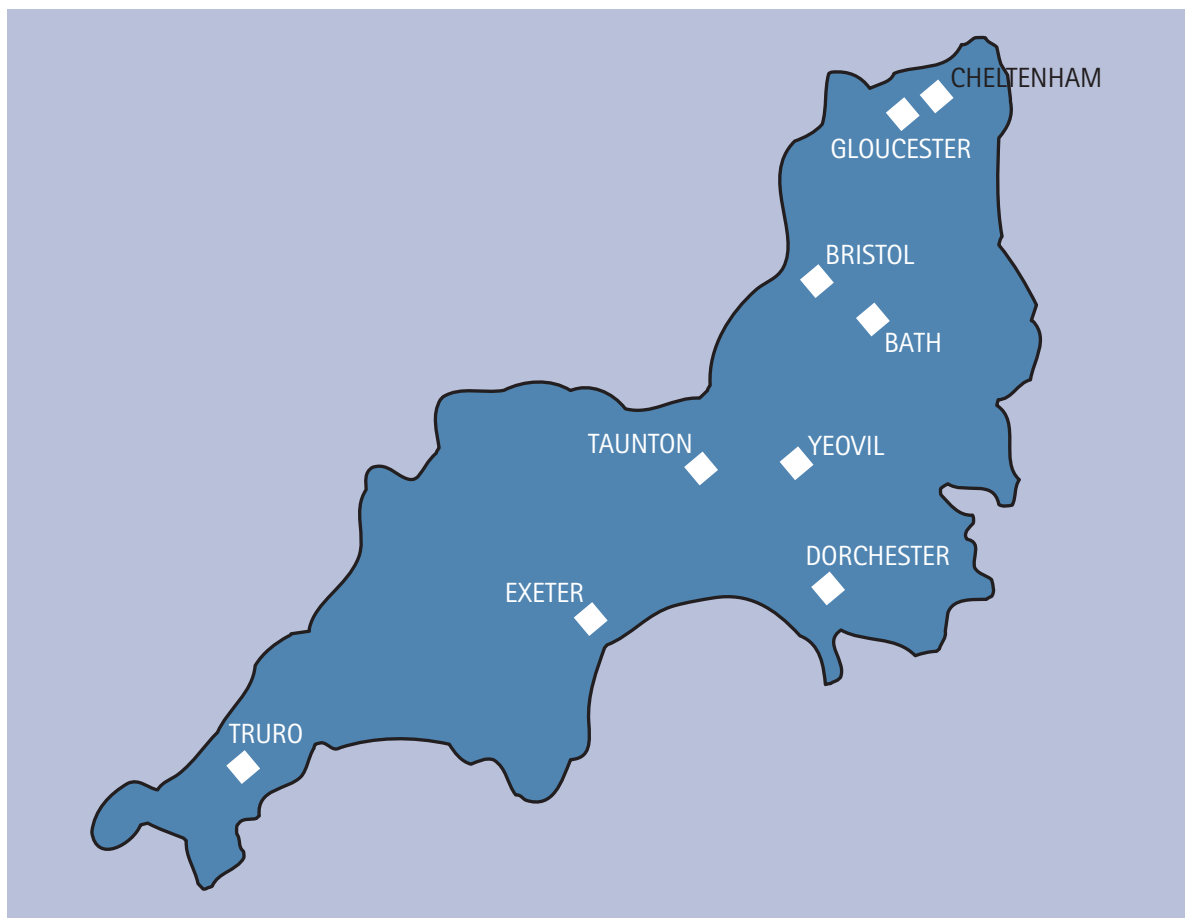

Fig. 1 Trial sites for the RCT on orthodontic pain management in the South West of England showing the large geographical spread

\section{METHOD}

The work diaries of seven of the PIs together with the research hygienist, the nurse and the administrative assistant were completed over a four month period (1 September 2011 to 31 December 2011).

In most RCTs there is a variation in the time input necessary at different stages of the clinical trial. In this trial these can be categorised into the 'set up', 'recruitment' and 'completion' stages.

When setting up an RCT it is time intensive for the CI while gaining necessary funding and approvals. This is followed by a busy period for the PIs while gaining local approval for the trial and setting up local processes for recruitment.

The initial stages of recruitment can also be time consuming as the clinicians begin to develop their structure for: identifying patients; explaining the trial; gaining consent; completing the necessary paperwork; and carrying out the intervention. Following the early recruitment phase of just a few weeks, the time commitment tends to plateau as clinicians become familiar with the processes.

Towards the end of recruitment there is a second peak in activity which includes: completion of all the trial paperwork; data entry; data analysis and interpretation of results and preparation of papers for publication.
It is during the plateau of recruitment that our PIs and the supporting staff completed their work diaries, recording the duties carried out and the time taken to perform these duties. When PIs received help in recruitment by their orthodontic colleagues this time was also recorded.

\section{RESULTS}

During the four month period 98 patients were recruited into the trial. The overall time spent by all those involved in this clinical trial was 12,629 minutes, or 210 hours. Since 98 patients were recruited during the diary period this equated to an overall average time of 129 minutes per patient. Of these 129 minutes, 19 minutes were clinical time that is, during orthodontic outpatient clinics, and 110 minutes were non-clinical time that is, out with clinics or the time spent by the supporting administrative staff (research hygienist and administrative assistant (Table 1).

There was considerable variation in the overall time commitment between recruiting centres ranging from 225 minutes to 5,807 minutes over the four month period. The clinical impact of recruiting patients at each centre ranged from 60 minutes (an average of 3 minutes per week) to 725 minutes (an average of 40 minutes per week). This reflects a difference in the number of patients recruited between the 


\begin{tabular}{|c|c|c|c|c|c|}
\hline Centre & $\begin{array}{l}\text { Total clinical time } \\
\text { (minutes) }\end{array}$ & $\begin{array}{l}\text { Total non-clinical } \\
\text { time (minutes) }\end{array}$ & $\begin{array}{l}\text { Number of } \\
\text { patients recruited }\end{array}$ & $\begin{array}{l}\text { Average clinical time per } \\
\text { patient recruited (minutes) }\end{array}$ & $\begin{array}{l}\text { Average non-clinical time per } \\
\text { patient recruited (minutes) }\end{array}$ \\
\hline Centre 1 & 340 & 491 & 26 & 13 & 19 \\
\hline Centre 2 & 160 & 4,395 & 12 & 13 & 366 \\
\hline Centre 3 & 725 & 5,082 & 39 & 19 & 130 \\
\hline Centre 4 & 60 & 245 & 1 & 60 & 245 \\
\hline Centre 5 & 195 & 30 & 8 & 24 & 4 \\
\hline Centre 6 & 221 & 244 & 8 & 28 & 30 \\
\hline Centre 7 & 134 & 307 & 4 & 33 & 77 \\
\hline Total & 1835 & 10794 & 98 & 19 & 110 \\
\hline
\end{tabular}

centres during the four months. However, it is the amount of non-clinical time spent which shows the most variation between centres, ranging from 30 minutes (an average of 1.6 minutes per week) to 5,082 minutes (an average of 282 minutes per week). This greater variation reflects the differences in administrative responsibilities between centres; the latter figure is from the main administrative centre and includes time spent on data entry as well as the local day-to-day administration carried out by all centres.

\section{DISCUSSION}

When planning a clinical research study it is often difficult for the CI to accurately determine the necessary time and resources that will be required to complete a clinical trial. This is particularly difficult in the case of a multicentre RCT where research support may vary from site to site. In this clinical trial the PIs were consultant orthodontists with full clinical caseloads. Recruitment was carried out in outpatient orthodontic clinics and the clinical impact ranged from an average 3 minutes per week at the centre recruiting least patients to 40 minutes per week at the centre recruiting most patients. These figures are specific to our trial protocol and time spent in recruitment will vary from clinical trial to clinical trial. More complicated protocols may require greater amounts of clinical time to explain the research aims to patients, check eligibility, recruit subjects or carry out the actual intervention, whereas less complicated protocols may require less time. Nevertheless it is hoped that the results of this survey can provide some baseline information to investigators planning an orthodontic clinical trial and help in the planning of resources. This information may also be of value to researchers in other dental disciplines and medical specialties when planning clinical trials.

Furthermore, when a consultant expresses an interest in becoming involved in a clinical trial, managers often require information about what will be involved. In particular they may seek reassurance that research activity will not have a significant impact on clinical activity. This survey shows that the impact on clinical care for this clinical trial was 19 minutes for each patient recruited, although no clinics were adjusted by PIs in this trial to accommodate the time taken for recruitment.

An important point to consider is that this clinical trial was accepted for inclusion within the UK Clinical Research Network (UKCRN) portfolio database making it eligible for NHS service support costs. For the 98 patients over the four month diary period this interventional clinical trial attracted service support costs in the region of $£ 145,500$. This funding is not directly allocated to researchers but to the regional comprehensive research networks and then to Trust R\&D departments. At four centres the PIs accessed a small amount of funds or support from their local R\&D departments. However, the level of funding accessed by the PIs was minimal compared to the service support costs generated by the clinical trial and recruitment may have been improved by accessing these funds in a more organised manner during the trial.

Of the seven PIs who completed work diaries only three had their research responsibilities recognised as part of their job plan and none had any specific time allocated within their job plan for research duties. When we consider that on average 110 minutes of time was needed to support the recruitment of each patient (almost six times as much as the clinical time) this is a considerable commitment for the clinicians involved, especially since many of the tasks were carried out in their own time or somehow included within the working week. These tasks were as diverse as telephoning patients to remind them to return their pain questionnaires to carrying out photocopying.

Good communication between researchers is vital to the success of a clinical trial and face-to-face meetings become more difficult when multiple centres are involved. In this trial, as the recruiting centres were spread over a wide geographical area, much of the initial communication around the trial was carried out by e-mail. Teleconferences were also organised, but because of clinical commitments these were scheduled in the evenings. Not all communication was possible through these means, especially at the early stages of the trial planning process and so face-to-face meetings were also organised. These took place on Sunday mornings in Taunton, which was geographically the most convenient centre of the region for the research team. Each of these meetings lasted for two to three hours. After the initial set up meetings, progress meetings were then held approximately every six months throughout the trial, again on Sunday mornings in Taunton. Although each meeting only lasted two to three hours, some PIs had 
return journey times of between one and a half and five hours per meeting due to the geographical spread within the region. This highlights the additional time commitment that researchers may have to make outside of their normal working week.

By quantifying the time spent in the recruitment phase of a randomised controlled trial it is hoped that clinicians considering becoming a PI will be more informed about the additional workload. There will be an impact on their clinics, which will vary with the complexity of the trial and the number of patients recruited. Perhaps more significant though is the time spent on administrative tasks around the trial. In this trial this was almost six times the amount of clinical time. However, despite the considerable time commitment, all of the participating PIs in this trial reported the overall experience of being involved in a clinical trial to be positive. On a personal level the PIs enhanced their research skills and also improved relationships with other local orthodontic consultants and academics involved in the trial. This involvement also had a wider positive impact at the recruiting centres. An improved understanding of the research process and being actively involved in recruitment was a valuable experience for trainees. Other clinicians, nursing and administrative staff also became interested in the research process and the possible research outcome. Many patients were excited about being involved in a clinical trial and were interested in how their contribution could influence advice given to future orthodontic patients. At many of the recruiting centres the profile of the orthodontic department within the Trust was also raised through participation in the research and inclusion in the UKCRN portfolio.

\section{CONCLUSION}

This study quantifies the time spent recruiting patients to a multicentre RCT. On average PIs spent 19 minutes of clinical time for each patient recruited and a further 110 minutes of supporting administrative work.

We would like to thank Hazel Donoghue, Medical Secretary, Dorset County Hospital; Jeanine Cunningham, Dental Nurse RUH NHS Trust Bath and the South West Research Design Service for their support.

1. O'Brien $\mathrm{K}$, Wright J, Conboy F et al. Effectiveness of early orthodontic treatment with the Twin-block appliance: a multicenter, randomized, controlled trial. Part 1: dental and skeletal effects. Am J Orthod Dentofacial Orthop 2003; 124: 234-243.

2. Bradley R L, Ellis P E, Thomas P, Bellis H, Ireland A J, Sandy J R. A randomized clinical trial comparing the efficacy of ibuprofen and paracetamol in the control of orthodontic pain. Am J Orthod Dentofacia Orthop 2007: 132: 511-517.

3. Mandall N, DiBiase A, Littlewood S et al. Is early Class III protraction facemask treatment effective? A multicentre, randomized, controlled trial: 15-month follow-up. J Orthod 2010; 37: 149-161.

4. DiBiase A T, Nasr I H, Scott P, Cobourne M T. Duration of treatment and occlusal outcome using Damon3 self-ligated and conventional orthodontic bracket systems in extraction patients: a prospective randomized clinical trial. Am J Orthod Dentofacial Orthop 2011; 139: e111-e116.

5. Cunningham S, Bearn D, Benson P et al. In search of the sample: recent experiences of a trial team in orthodontics. Contemp Clin Trials 2011: 32: $530-534$.

6. Hichens L P, Sandy J R, Rowland H N et al. Practical aspects to undertaking research in the primary care setting: experience from two studies. J Orthod 2005: 32: 262-268. 